\title{
Programa de Educação pelo Trabalho (PET) em Saúde: articulação entre formação e integração ensino-serviço
}

\author{
Program of Education through Work (PET) in Healthcare: linkage between qualification and \\ integration of teaching and work \\ Programa de Educación por medio del Trabajo (PET) en Salud: vinculación de formación e \\ integración enseñanza - servicio
}

\author{
Jaime Everardo Platner Cezario'; Donizete Vago Daher"; Marja Ferreira Soares Nolasco ${ }^{\text {III }}$
}

\begin{abstract}
RESUMO: Objetivou-se identificar as percepções de alunos bolsistas e preceptores do Programa de Educação pelo Trabalho em Saúde (PET-Saúde) sobre a integração ensino-serviço (IES) no processo de formação e analisar a contribuição deste Programa para a formação de profissionais de saúde. A abordagem é qualitativa e descritiva, com dados coletados entre 2010 e 2012, por meio de observações e entrevistas, cujos cenários foram as unidades de saúde do Município de Niterói. Participaram 16 sujeitos, entre bolsistas e preceptores do Programa. A análise temática produziu duas categorias: A essencialidade da IES na formação em saúde; e A formação em saúde e o desafiador processo de construção da IES. Encontros e desencontros entre teoria e prática foram relatados como se a dissociação fizesse parte de uma cultura instituída. Concluiu-se que as atividades do Programa geraram possibilidades de construção e reconstrução de saberes e práticas.

Palavras-Chave: Formação; PET-saúde; serviços de integração docente assistencial; enfermagem.
\end{abstract}

\begin{abstract}
The aim of this qualitative, descriptive study was to identify how scholarship students and tutors of the Program of Education through Work (PET) in Health perceived the integration between teaching and work (IES) in the training process, and to examine how this program contributed to the health professionals' training. Data were collected between 2010 and 2012 by observation and interviews of the 16 participating tutors and scholarship students at healthcare facilities in Niterói. Our thematic analysis generated two categories: IES is essential to training in healthcare; and training in healthcare and the challenging process of building IES. Matches and mismatches between practice and theory were reported as if the dissociation formed part of an established culture. It was concluded that the program activities offered opportunities for building and rebuilding knowledge and practices. Keywords: Training; PET-health; teaching-healthcare integration services; nursing.

RESUMEN: El objetivo fue identificar las percepciones de estudiantes becarios y preceptores del Programa de Educación para el Trabajo en Salud (PET-Salud) sobre la integración de enseñanza y servicio (IES) en el proceso de formación y analizar la contribución de este programa en la formación de los profesionales de salud. El abordaje es cualitativo y descriptivo, con datos recolectados entre 2010 y 2012, a través de observaciones y entrevistas, en unidades de salud del municipio de la ciudad de Niterói. El estudio tuvo 16 participantes: becarios y preceptores del programa. El análisis temático produjo dos categorías: La esencialidad de la IES en la formación en salud; y la Formación en salud y el desafiador proceso de construcción de la IES. Acuerdos y desacuerdos entre teoría y práctica han sido reportados como si la disociación formara parte de una cultura establecida. Se concluye que las actividades del Programa generaron posibilidades de construcción y reconstrucción de conocimientos y prácticas. Palabras Clave: Formación; PET-salud; servicios de integración asistencial de profesores; enfermería.
\end{abstract}

\section{INTRODUÇÃO}

Estudos sobre o processo de formação de profissionais da saúde vêm ocupando expressivo espaço em debates, em especial após a implantação do Sistema Único de Saúde (SUS), das Diretrizes Curriculares Nacionais (DCN) e após a implementação da Estratégia de Saúde da Família (ESF). Estes investimentos dos Ministérios da Educação e da Saúde foram instituí- dos com vistas a redirecionar o modelo de atenção à saúde vigente e reorientar as práticas de formação em saúde. Assim, ao nos debruçarmos sobre as reflexões promovidas pelos cursos da área da saúde, constatamos que é recorrente, tanto por parte de docentes, quanto por parte de acadêmicos, uma postura de desconexão com o ambiente em que realizam a formação prática.

'Enfermeiro. Mestre em Formação Pedagógica em Saúde pelo Mestrado Profissional em Ensino na Saúde da Escola de Enfermagem Aurora de Afonso Costa, Universidade Federal Fluminense. Niterói, Rio de Janeiro, Brasil. E-mail: shovegan@yahoo.com.br.

IEnfermeira. Professora Associada da Escola de Enfermagem Aurora de Afonso Costa, Universidade Federal Fluminense. Niterói, Rio de Janeiro, Brasil. E-mail: donizete@predialnet.com.br.

IIIEnfermeira. Mestranda do Programa Acadêmico em Ciências do Cuidado em Saúde da Escola de Enfermagem Aurora de Afonso Costa, Universidade Federal Fluminense. Niterói, Rio de Janeiro, Brasil. E-mail: marjafs@hotmail.com. 
Ao que parece, a unidade primária de saúde (UPS), a rede de assistência que compõe o SUS, é entendida como espaço para ser apresentado e observado sem a interconexão necessária ao processo ensino-aprendizagem. Ao mesmo tempo há, por conta do profissional do serviço, resistência, negação em fazer parte ou compor este processo de formação.

Nesse sentido, detectamos distanciamentos de todos os envolvidos, o que contribuiu para a eleição do objeto integração ensino-serviço (IES) na formação do profissional de saúde por meio do Programa de Educação pelo Trabalho para a Saúde (PET-Saúde).

Este estudo traz subsídios tanto para o ensino como para os serviços de saúde, na medida em que conclama a todos os envolvidos a reorientação de seus saberes e fazeres.

Elegemos como questão: Qual a percepção de alunos bolsistas e preceptores, participantes do PET-Saúde, sobre o processo de formação e as possibilidades de IES?

Os objetivos do estudo são: identificar as percepções de alunos bolsistas e preceptores do Projeto PET-Saúde sobre a IES no processo de formação; e analisar a contribuição do PET-Saúde no processo de formação de profissionais de saúde.

\section{REVISÃO DE LITERATURA}

O quadro de desarticulação entre teoria e prática nos processos de formação em saúde é uma realidade no Brasil, podendo ser amenizado pela efetiva IES, unindo docentes, estudantes e profissionais de saúde com o foco central no usuário e, também, com investimento na sensibilização dos participantes inseridos nos cenários em que se desenvolvem os cuidados e o processo de ensino-aprendizagem ${ }^{1}$.

O tema IES indica que, mesmo diante de propostas inovadoras que defendam uma formação voltada para a implementação dos princípios e diretrizes do SUS, vivenciamos modos de formar profissionais de saúde geradores de práticas descontextualizadas, norteadas por fragmentação de saberes e fazeres ${ }^{1-3}$.

A parceria entre o ensino e o serviço deverá consistir em um processo facilitador entre trabalho, educação e saúde, procurando refletir sobre a permanente dicotomia entre pensar e fazer, entre geral e específico, integrando ações e habilidades teóricas e práticas ${ }^{4: 378}$.

A Secretaria de Gestão do Trabalho e da Educação na Saúde (SGTES) instituiu, em 2007, o PET-Saúde. Concebido como possibilidade de ponte de ligação entre o ensino-serviço-comunidade, o PET-Saúde visa formar grupos de ensino-aprendizagem orientados por tutores e alocados em áreas consideradas de especial atenção para o SUS, servindo, assim, como ferramenta para a aproximação das políticas públicas de saúde e a prática dos serviços.
Debates no Brasil têm produzido esforços para o câmbio nos processos de formação profissional, consubstanciados politicamente no surgimento de diferentes iniciativas, cabendo destaque à articulação levada a cabo pelos Ministérios da Saúde e da Educação, manifesta na elaboração de ações como o Programa de Incentivo às Mudanças Curriculares nas Escolas Médicas (PROMED) e o Programa Nacional de Reorientação da Formação Profissional em Saúde (PROSAÚDE) e PET-Saúde. Todas as ações desenvolvidas pelos envolvidos nos Programas devem resultar em um perfil profissional com habilidades para identificar, minorar e/ou resolver os problemas de saúde ${ }^{5-7}$.

A Universidade Federal Fluminense (UFF) e a Fundação Municipal de Saúde (FMS-Niterói) reafirmaram a parceria objetivando

[...] fortalecer os mecanismos de cooperação entre gestores da rede local de saúde e a UFF, ampliar os cenários de aprendizagem; intensificar, na formação, a abordagem integral do processo saúdedoença e da promoção da saúde $[. . .]^{8: 436}$.

Desse modo, é importante compreender como está sendo desenvolvida a formação profissional em saúde frente às novas demandas e discussões teóricas e práticas no âmbito do SUS. A busca pela superação da dicotomia entre teoria e prática, entre o pensar e o fazer em saúde, tem sido objeto de reflexão de muitos estudos buscando a integração na perspectiva de avançar para um SUS mais eficiente e resolutivo das necessidades individuais e coletivas ${ }^{9,10}$.

Nesse contexto, o PET-Saúde busca a relevância da discussão sobre formação e IES pela possibilidade da aproximação da instituição de formação com a realidade do SUS. Sua intenção é conduzir a uma reflexão sobre as novas propostas pedagógicas de formação, que buscam o equilíbrio entre excelência técnica e relevância social, estes entendidos como espaços que contribuem para o movimento de mudança curricular, em modelos pedagógicos mais interativos, na adoção de metodologias ativas de ensino-aprendizagem, centradas no aluno como sujeito da aprendizagem e no professor, como facilitador do processo de construção de conhecimento ${ }^{11}$.

\section{Metodologia}

A abordagem qualitativa e descritiva foi a que melhor se adequou ao nosso estudo. A coleta de dados ocorreu por meio de trabalho de campo realizado em dois momentos: observação participante e entrevista semiestruturada individual. A observação participante aconteceu em diferentes momentos, como, por exemplo, quando um dos pesquisadores atuava em campo, ou quando tinha a oportunidade de observar o cotidiano dos demais preceptores e dos bolsistas no serviço, durante a efetivação do Projeto PET-Saúde. As entrevistas, depois de agendadas, aconteceram em 
espaços eleitos pelos participantes, sendo gravadas, transcritas e analisadas por meio de separação por temas em três etapas: pré-análise, exploração do material e tratamento dos resultados obtidos, e interpretação ${ }^{12}$.

Os sujeitos da pesquisa, eleitos de forma aleatória, foram nove alunos graduandos da área de saúde, a saber: dos cursos de enfermagem, medicina, nutrição, farmácia, odontologia e educação física todos bolsistas do grupo PET-Saúde/UFF. Além dos discentes, participaram sete preceptores, profissionais dos diferentes serviços de atenção básica de saúde de Niterói-RJ , constituindo cenário deste estudo.

Os sujeitos concordaram em participar, assinando o Termo de Consentimento Livre e Esclarecido (TCLE), sendo-lhes assegurado o sigilo quanto às informações prestadas e à sua identidade, atendendo, desta forma, à Resolução n ${ }^{\circ}$ 466/2012, do Conselho Nacional de Saúde. Nesse sentido, como forma de manter o anonimato dos participantes, os preceptores foram identificados com a letra $P$, acompanhada por um número que seguiu a sequência de entrevistados, e os bolsistas, com a letra $B$, seguida de um número e também na ordem de entrevista.

Como critério de inclusão, foi estabelecido: alunos regularmente matriculados em cursos de graduação na área de Saúde, bolsistas do PET-Saúde, entre os anos de 2010 e 2012, que concordassem em participar, assinando o TCLE; profissionais de nível superior da rede pública de saúde do Município de Niterói, que participaram como preceptores/bolsistas do PET-Saúde, cientes do objetivo da pesquisa e que assinassem o TCLE. Para critérios de exclusão, foram adotados os seguintes quesitos: alunos de graduação dos cursos da área de saúde e profissionais do município de Niterói que participaram do PET-Saúde por período menor que três meses e aqueles integrantes do Programa que discordassem em participar do estudo. Pesquisa aprovada com o n ${ }^{\circ} 184.881$ pelo Comitê de Ética em Pesquisa da Faculdade de Medicina e do Hospital Universitário Antônio Pedro/UFF.

\section{Resultados e Discussão}

Com o propósito de atender aos objetivos, apresentamos e analisamos os resultados por meio de dois núcleos temáticos descritos a seguir.

\section{A essencialidade da IES na formação em saúde}

Ao serem questionados sobre como compreendiam a IES, os participantes destacaram a sua essencialidade:

Possui uma relevância muito grande, ajuda a diminuir distâncias. Porque eu tive a oportunidade de pertencer a uma instituição que tinha hospital próprio e nós ficávamos muito focadas com uma única instituição. Então, em meu primeiro emprego, início de vida profissional foi um pouco assustador. Eu não tinha mais o professor. Hoje, 22 anos depois, eu vejo como é importante essa integração, isso tem que começar desde o primeiro momento que nós pisamos na instituição de ensino. Ela é essencial. (P3)

Eu acho que o serviço e o ensino estão completamente interligados. É essencial. Não existe, na verdade, o serviço sem você ter o ensino que é a parte teórica, que fundamenta. Mas que, assim, nós não aprendemos só a teoria. Às vezes, falta a parte prática. No PET, nós conseguíamos fazer essa relação pelo trabalho com o profissional. (B4)

\section{A IES é entendida como}

[...] espaços onde se dá o diálogo entre o trabalho e a educação e estes assumem lugar privilegiado para a percepção que o estudante vai desenvolvendo acerca do outro no cotidiano do cuidado ${ }^{1: 358}$.

Assim, são espaços de formação permanente, de construção da identidade profissional e de cidadania, nos quais os profissionais do serviço, docentes, usuários e os estudantes vão construindo e estabelecendo seus papéis sociais na confluência de seus saberes, modos de ser e de ver o mundo.

Grande crédito tem sido depositado na IES, como instrumento de passagem da atual concepção, tal que "as principais consequências desse modelo de formação são: a produção fragmentada do cuidado e a baixa capacidade de reflexão sobre a prática profissional"13:520, para um processo que contraponha a situação vigente. Assim, buscamos apresentar uma abordagem temático-conceitual para o desenvolvimento e fortalecimento do pensamento crítico na formação dos profissionais de saúde.

O PET-Saúde foi defendido pelos participantes como uma relevante estratégia para se trabalhar a IES. Nesse sentido, ao serem analisadas as possibilidades do Programa, destacamos que este pode ser entendido como "uma estratégia de ação capaz de contribuir para melhor articulação entre a academia e os serviços de saúde"14:545.

Ressaltamos que os

[...] cenários de prática para formação profissional devem ser diversificados e aglutinadores de equipamentos educacionais e comunitários, para além dos equipamentos de saúde, favorecendo uma maior aproximação entre os estudantes e a realidade dos serviços $^{15: 41-42}$.

\section{A formação e o processo de construção da IES}

Estimulados a refletirem sobre a formação dos profissionais de saúde e a vivência deste processo em seus cenários de prática, os participantes do estudo se expressaram de diferentes formas:

Tenho percebido que há uma preocupação muito grande em formar, mas não está tendo uma preocupação muito mais importante que é com a qualidade dessa formação. E nós sabemos que é importante. Você não vê integração entre os profissionais. E essa integração deveria ser muito mais trabalhada. (P3)

A parte onde eu vi mais essa integração da teoria com a prática foi no PET. Quando você entra no 
serviço, num estágio, eu não vejo tão forte essa integração, daquele que acompanha a gente, no campo, no estágio curricular com o professor. Já no PET não, tivemos contato com o enfermeiro, com o médico direto. Tudo foi muito mais forte, essa relação, essa integração com todo mundo. Tanto que eu amei o PET por isso. (B1)

Reconhecendo que há limitações no processo de formação dos profissionais de saúde, em especial a tênue participação dos serviços neste processo, e que a direção a ser tomada na busca da excelência técnica e científica passa pela valorização das percepções e pelos saberes e experiências acumulados pelos profissionais dos serviços, este que vive a realidade no seu dia a dia, ressaltamos que:

[...] o momento atual requer ajustes e releituras dos profissionais e das instituições para eleger estratégias e modelos de renovação coerentes com o contexto. Nesse sentido, [...] a compreensão de que é necessário [...] ações no campo educacional que possibilitem um avanço no conhecimento do trabalho em saúde, reconhecendo e valorizando o potencial formativo do trabalho ${ }^{16: 176}$.

Cada profissional de saúde que vivencia os serviços, dele fazendo parte, e que for ouvido acerca de sua formação acadêmica e prática, imprimirá sua própria concepção sobre IES. Ao revisitar o seu processo de apreensão do conhecimento e com aquele que se depara hoje, ele poderá antever e indicar saídas, mesmo entendendo-as como desafiadoras.

Para intervir na realidade,

[...] é necessário que os setores da educação e da saúde estabeleçam uma estreita e permanente parceria interinstitucional, objetivando desenvolver ações conjuntas e articuladas para a elaboração e a construção de uma proposta educacional que conjugue os conhecimentos produzidos e acumulados pelas duas áreas. [... $]^{4: 377}$.

Os encontros e desencontros entre a teoria e a prática, vivenciados pelos participantes, bolsistas e profissionais, em seus processos de trabalho, foram relatados com naturalidade e muita certeza, como se a dissociação presente fizesse parte de uma cultura, já formalizada como normal, cotidiana.

Assim, é necessário rever a formação com vista a uma nova práxis, a qual transite entre as dimensões cuidadora, gerencial, educadora e de investigação do processo de trabalho da enfermagem, para que ele assuma seu papel de articulador no sistema, nos serviços e na assistência à saúde, na perspectiva da integralidade, da integração do ensino-serviço ${ }^{17}$.

A aproximação entre os diversos níveis de gestão é uma das ferramentas que favorece a IES e a formação acadêmica, merecendo ser constantemente estimulada. Tal atitude contribui para a redução ou, quem sabe, a eliminação dos estranhamentos que limitam a articulação das propostas de integração.

\section{Conclusão}

Este estudo possibilitou o reconhecimento da percepção de alunos bolsistas e preceptores participantes do Programa PET-Saúde sobre a IES. Da análise dos depoimentos, emergiram duas categorias temáticas: A essencialidade da IES na formação em saúde e A formação em saúde e o processo de construção da IES.

O PET-Saúde apresenta-se como espaço concreto para se trabalhar a formação e a IES, além de favorecer a inserção dos participantes no cotidiano da comunidade onde está sendo desenvolvido o projeto. Dessa forma, o que se constrói, fruto desta simbiose, é a possibilidade do surgimento de um profissional em sintonia com as necessidades de saúde da população e com a execução de ações que fortalecem o sistema de saúde brasileiro.

A limitação do estudo ocorreu pela reduzida adesão dos participantes do PET-Saúde quando do convite para a pesquisa, fazendo crer que o objetivo da pesquisa foi visto como estratégia pontual, cujas ações de saúde em parceria se findam com o término do Programa. Concluímos que a IES é um processo em construção que precisa de programas similares ao PET-Saúde para a sua efetiva consolidação.

\section{REFERÊNCIAS}

1.Albuquerque VS, Giffin KM. A integração ensino-serviço no contexto dos processos de mudança na formação superior dos profissionais da saúde. Rev bras educ med. 2008; 32:356-62.

2.Ceccim RB, Feuerwerker LCMO. Quadrilátero da formação para a área da saúde: ensino, gestão, atenção e controle social. Physis: Rev saude coletiva. 2004; 14:41-65.

3.Santos DN, Killinger CL, organizadoras. Aprender fazendo: a interdisciplinaridade na formação em saúde coletiva. Salvador(BA): EDUFBA; 2011.

4.Amâncio Filho A. Dilemas e desafios da formação profissional em saúde. Interface - Comunic, Saude, Educ. 2004; 8:375-80.

5.Siqueira BR, Gomes AP, Albuquerque VS, Cavalcanti FOL, Cotta RMM. Educação e competências para o SUS: é possível pensar alternativas à(s) lógica(s) do capitalismo tardio? Ciênc saúde coletiva. 2013; 18:159-70.

6.Ferraz L. O PET-Saúde e sua Interlocução com o Pró-Saúde a partir da pesquisa: o relato dessa experiência. Rev bras educ med. 2012; 36:166-71.

7.Haddad AE, Brenelli SL, Passarella TM, Ribeiro TCV. Política nacional de educação na saúde. Rev baiana de Saúde Públ. 2008; 32:98-114.

8.Abrahão AL, Cordeiro BC, Marques D, Daher DV, Teixeira GHMC, Monteiro KA et al. A pesquisa como dispositivo para o exercício no PET-Saúde UFF/FMS Niterói. Rev bras educ med. 2011; 35:435-40.

9.Pimentel MRAR, David HMSL. Formação crítica de 
enfermeiros: repercussões na Universidade do Estado do Rio de Janeiro. Rev enferm UERJ. 2013; 21:247-53. 10.Pires AS, Souza NVDO, Penna LHG, Tavares KFA, D'Oliveira CAFB, Almeida CM. A formação de enfermagem na graduação: uma revisão integrativa da literatura. Rev enferm UERJ. 2014; 22:705-11.

11.Feuerwerker LCM, Sena RR. A contribution to the movement for change in professional healthcare education: an assessment of the UNI experiences, Interface - Comunic, Saude, Educ. 2002; 6:37-50.

12.Minayo MC, Deslandes SF, Cruz Neto O, Gomes R. Pesquisa social: teoria, método e criatividade. Petrópolis (RJ): Vozes; 2003.

13.Albuquerque VS, Giffin KM. Globalização capitalista e formação profissional em saúde: uma agenda necessária ao ensino superior. Trab educ saúde. 2009; 6:519-37. 14.Morais FRR, Jales ML, Silva MJCS, Fernandes SF. A importância do PET-Saúde para a formação acadêmica do enfermeiro. Trab educ saúde. 2012; 10:541-51.

15.Lima JO. Uma estratégia para articulação ensino-serviço no SUS-BA: A rede de integração da educação e trabalho na saúde [dissertação de mestrado]. Rio de Janeiro: Escola Nacional de Saúde Pública; 2009.

16.Ministério da Saúde (Br). Secretaria de Gestão do Trabalho e da Educação na Saúde. Rev - SUS Brasil. Brasília(DF): Ministério da Saúde; 2004.

17.Santos JLG, Pestana AL, Guerrero P, Meirelles BSH, Erdmann AL. Práticas de enfermeiros na gerência do cuidado em enfermagem e saúde: revisão integrativa. Rev Bras Enferm. 2013; 66:257-63. 